\title{
Autopercepção das condições bucais em uma população de idosos da Cidade de Montes Claros, Minas Gerais, Brasil
}

Self-Perception of oral conditions in a population of community-dwelling elderly in Montes

Claros City, State of Minas Gerais, Brazil

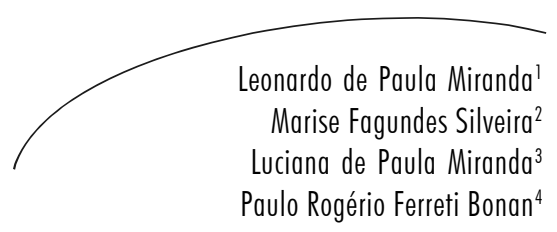

Resumo

O objetivo deste trabalho foi investigar a autopercepção das condições bucais em uma população de idosos não-institucionalizados da cidade de Montes Claros. A pesquisa objetivou ainda verificar a associação existente entre a autopercepção e as características sociodemográficas, necessidades odontológicas, condições sistêmicas e uso de medicações nos indivíduos pesquisados. Trata-se de um estudo descritivo transversal com uma amostra do tipo aleatória simples $(n=204)$. A autopercepção foi avaliada através do Índice de Determinação da Saúde Bucal Geriátrica (GOHAI). De acordo com os resultados obtidos, $53,9 \%$ dos idosos eram do gênero feminino; $53,9 \%$ não eram casados; $57,8 \%$ possuíam idade entre 60 e 69 anos; $84,9 \%$ não eram brancos; $69,1 \%$ eram analfabetos; $56,4 \%$ tinham renda per capita $>1 / 2$ salário mínimo; $59,8 \%$ foram ao dentista há três anos ou mais; $52,9 \%$ realizaram a última consulta no serviço público; $63,7 \%$ eram edêntulos; $67,2 \%$ usavam algum tipo de prótese; $77 \%$ necessitavam de prótese e $81,4 \%$ não possuíam alteração de tecido mole. Destes, $31,9 \%$ apresentavam doença periodontal; o CPO-D médio foi 29,39; 91,2\% apresentavam alguma doença sistêmica; $78,4 \%$ usavam medicação e a maior parte (36,3\%) autopercebeu a condição bucal como regular. $\mathrm{Na}$ análise multivariada, foi verificado que os idosos que necessitavam de algum tipo de prótese dentária e não possuíam cônjuge apresentaram maiores chances de ter uma baixa autopercepção das condições bucais. De maneira geral, percebeu-se que a maioria dos idosos possuía uma condição socioeconômica satisfatória; apresentava necessidades odontológicas normativas; tinha alguma doença sistêmica; usava medicação e autopercebeu a saúde bucal como regular ou ruim, condizente com a avaliação profissional.

\footnotetext{
Universidade Estadual de Montes Claros. Montes Claros, Minas Gerais, Brasil.

1 Programa de Pós-graduação em Ciências da Saúde, Mestrado Profissional em Cuidado Primário em Saúde.

2 Departamento de Matemática

Departamento de Enfermagem

Programa de Pós-graduação em Ciências da Saúde.
}

Correspondência / Correspondence

Paulo Rogério Ferreti Bonan

Rua Helena de Paula Fraga, 867, Major Prates

Cep: 39403-263 - Montes Claros, MG, Brasil

E-mail: pbonan@yahoo.com

Palavras-chave: Prestação de Assistência Odontológica. Autoprecepção. Saúde do idoso. Autoavaliação. Idoso. Saúde bucal. 


\section{Abstract}

The aim of this study was to investigate the self-perception of oral health in a population of community-dwelling elderly in Montes Claros city, MG. The research aimed further to investigate the existing association between selfperception and sociodemographic characteristics, dental needs, systemic conditions and medication use in those individuals. This is a cross-sectional study with a simple random sample $(\mathrm{n}=204)$. The self-perception was assessed using the Geriatric Oral Health Assessment Index (GOHAI). According to the results, $53.9 \%$ of the elderly were female, $53.9 \%$ were not married, $57.8 \%$ were aged between 60 and 69 years old, $84.9 \%$ were not white, $69.1 \%$ were illiterate, $56.4 \%$ had an monthly household income $>1 / 2$ minimum wage, $59.8 \%$ went to the dentist for 3 years of more, $52.9 \%$ had a last consultation in the public service, $63.7 \%$ were edentulous, $67.2 \%$ used some type of dental prosthesis, $77 \%$ needed dental prosthetic. Of them, $81.4 \%$ had no alteration in soft tissue, $31.9 \%$ had periodontal disease, the CPO-D medium was $29,39,91.2 \%$ had systemic disease, $78.4 \%$ used medication and most (36.3\%) self-perceived oral health as a regular. In the multivariate analysis, it was found that the older people that needed some kind of dental prosthesis and had no spouse were more likely to have a low selfperception of oral conditions. Overall, it was observed that most elderly people had a satisfactory socioeconomic status, had dental needs, had any systemic disease, used medication and self-perceived oral health as fair or poor, consistent with the professional assessment.
Key words: Delivery of Health Care. Self

Concept. Health of the Elderly. Self-assessment. Elderly. Oral health.

\section{INTRODUÇÃOO}

Com o aumento da expectativa de vida da população e, consequentemente, da parcela de idosos, o conceito de qualidade de vida torna-se importante. A saúde bucal tem um papel relevante na qualidade de vida de indivíduos da terceira idade, uma vez que seu comprometimento pode afetar negativamente o nível nutricional, o bemestar físico e mental, bem como diminuir o prazer de uma vida social ativa. ${ }^{1}$

Verifica-se que os idosos normalmente fazem uso de múltiplas medicações para controle de problemas crônicos, ${ }^{2}$ ressaltando-se o consumo dos seguintes grupos de medicamentos: cardiovasculares, analgésicos, sedativos e tranquilizantes. ${ }^{3}$ Essas drogas geralmente originam efeitos colaterais, com repercussão na cavidade bucal como xerostomia, diminuição na produção da amilase salivar, alterações no paladar, sialorreia, hiperplasia gengival, aumento no número de cáries, doenças periodontais, candidíase e estomatites. $^{4,5}$ Os medicamentos antihipertensivos, antidepressivos, ansiolíticos, anticolinérgicos e anti-histamínicos estão intimamente ligados à xerostomia, que é o efeito colateral mais prevalente nesses indivíduos. ${ }^{5}$

Dentre as alterações bucais mais prevalentes na população idosa, destacam-se as lesões cariosas, edentulismo, periodontopatias, desgastes dentais, lesões em tecidos moles, desordens têmporomandibulares, dores orafaciais, problemas de oclusão e câncer bucal. ${ }^{6}$ Seguindo uma tendência, os estudos epidemiológicos em saúde bucal, que tradicionalmente enfocavam apenas as condições e alterações bucais dos indivíduos, estão cada vez mais sendo complementados com dados relativos às características socioeconômicas das pessoas, devido à influência dessas características nas condições de saúde da população, ${ }^{7}$ proporcionando, assim, uma dimensão mais ampla às pesquisas odontológicas.

Silva \& Castellanos-Fernandes ${ }^{8}$ enfatizam que é essencial entender como a pessoa percebe sua condição bucal, pois seu comportamento é condicionado pela importância dada a ela. A autopercepção de saúde mensura, de uma maneira 
muita mais global, o estado de saúde do indivíduo, incorporando aspectos da saúde cognitiva e emocional, como também da saúde física. $^{9}$

Estudos sobre a autopercepção da saúde bucal mostram estar ela relacionada a alguns fatores clínicos, como número de dentes cariados, perdidos ou restaurados, ${ }^{10} \mathrm{e}$ com fatores subjetivos, como sintomas das doenças e capacidade de a pessoa sorrir, falar ou mastigar sem problemas, ${ }^{11}$ além de ser também influenciada por fatores como classe social, idade, renda e sexo. ${ }^{12}$

O presente estudo teve como objetivo investigar a autopercepção das condições bucais em uma população de idosos nãoinstitucionalizados e cadastrados em três equipes da Estratégia de Saúde de Família da cidade de Montes Claros, Minas Gerais, Brasil. A pesquisa objetivou ainda verificar a associação existente entre a autopercepção e as características sociodemográficas, necessidades odontológicas, condições sistêmicas e uso de medicações nos indivíduos pesquisados.

\section{METODOLOGIA}

Neste estudo descritivo transversal de base populacional, foi avaliada a população idosa cadastrada na Estratégia de Saúde da Família do bairro Independência, localizado na zona leste da cidade de Montes Claros, Minas Gerais. O bairro conta com três equipes da Estratégia de Saúde da Família, onde há $100 \%$ de cobertura. Essas equipes englobam uma população adscrita total de 434 idosos $(\mathrm{N}=434)$.

Em relação ao tamanho da amostra, para efeito de cálculo, foi estimada uma prevalência de 50\% para as doenças bucais em uma população finita, um nível de significância $\alpha$ de $5 \%$ um intervalo de confiança de $95 \%$, resultando assim em uma amostra composta por 204 idosos $(\mathrm{n}=204)$. Para a seleção da amostra, foi utilizada a técnica de amostragem do tipo casual simples. Os agentes comunitários de saúde forneceram listas com os nomes (cada nome recebeu uma numeração) e endereços de todos os idosos cadastrados nas três Equipes da Estratégia Saúde da Família do bairro e, a partir dessa listagem, realizou-se um sorteio para selecionar os indivíduos participantes do estudo.

Os critérios de inclusão do estudo foram os seguintes: possuir idade mínima de 60 anos, ser cadastrado em uma das três equipes da Estratégia Saúde da Família e aceitar participar da pesquisa através da assinatura do termo de consentimento livre e esclarecido. Os idosos que apresentaram déficits cognitivos que poderiam dificultar ou impedir a transmissão de informações referentes às variáveis pesquisadas foram excluídos do estudo. O diagnóstico do déficit cognitivo foi feito através da utilização da versão em português do MiniExame do Estado Mental (MEEM), traduzida e modificada, ${ }^{13}$ considerando os pontos de corte 19 e 25, segundo a ausência (analfabetos) ou presença de instrução escolar formal prévia, respectivamente. Assim, foram considerados portadores de déficits cognitivos os idosos analfabetos que obtiveram um escore $\leq 19$ e os alfabetizados com escore $\leq 25 .{ }^{13}$ Foram excluídos ainda os idosos que não compareceram à unidade de saúde para realização da entrevista e exame clínico, após dois agendamentos consecutivos. Em relação às doenças sistêmicas que os idosos apresentavam, só foram registradas no instrumento de coleta de dados aquelas em que um diagnóstico prévio já havia sido estabelecido por um profissional de saúde e que foram relatadas pelo indivíduo no momento da entrevista.

A coleta de dados envolveu a realização de entrevistas estruturadas e exames clínicos odontológicos. Para a realização da entrevista, utilizou-se um questionário estruturado para colher informações referentes às características sociodemográficas, condições sistêmicas, uso de medicações e autopercepção das condições bucais (avaliada através do Índice de Determinação da Saúde Bucal Geriátrica - GOHAI). ${ }^{14}$

A definição dos itens do instrumento de coleta de dados, bem como a definição dos critérios a serem adotados na pesquisa seguiram, basicamente, aqueles adotados no Levantamento das Condições de Saúde 
Bucal da População Brasileira. ${ }^{15}$ Um estudo pré-teste envolvendo $5 \%$ da amostra foi realizado com o objetivo de testar a aplicabilidade do instrumento de coleta de dados. O examinador (próprio pesquisador) foi previamente calibrado (calibração intraexaminador), através do exame e reexame de $10 \%$ dos indivíduos, seguindo recomendação da OMS. ${ }^{16} \mathrm{O}$ teste Kappa foi utilizado para verificar o nível de concordância intra-examinador.

Os valores da concordância percentual e do teste Kappa foram os seguintes: CPI - concordância de 96\% (Padrão-ouro: SB Brasil 2000 - 95\%) ${ }^{15}$ e Kappa 0,86 (concordância excelente) ${ }^{17}$; PIP - concordância de 95\% (Padrão-ouro: SB Brasil $2000-95 \%)^{15}$ e Kappa 0,78 (concordância excelente) ${ }^{17}$; Cárie dentária/condição da coroa - concordância de $98 \%$ (Padrão-ouro: SB Brasil 2000 - 97,5\%) ${ }^{15}$ e Kappa 0,92 (concordância excelente) ${ }^{17}$; Cárie dentária/ condição da raiz - concordância de 97,6\% (Padrãoouro: SB Brasil 2000 - 97,5\%) $)^{15}$ e Kappa 0,90 (concordância excelente) ${ }^{17}$; Cárie dentária/ necessidade de tratamento - concordância de 97,6\% (Padrão-ouro: SB Brasil 2000 - 97,5\%) ${ }^{15}$ e Kappa 0,91 (concordância excelente) ${ }^{17}$; Uso e necessidade de prótese superior - concordância de $100 \%$ e Kappa 1,00; Uso e necessidade de prótese inferior concordância de 100\% e Kappa 1,00; Alterações de tecido mole - concordância de 100\% e Kappa 1,00.

Os exames clínicos foram realizados no consultório odontológico da Unidade de Saúde da Família Independência I e III, utilizando luz artificial, sonda exploradora $n^{\circ} 5$, espelho bucal plano, sonda periodontal desenvolvida pela OMS (Golgran ${ }^{\circledR}$ ), espátulas de madeira e equipamento de proteção individual. A anotação dos dados referentes aos exames clínicos foi feita por uma auxiliar de consultório dentário.

A coleta de dados ocorreu no período compreendido entre novembro de 2008 e maio de 2009. Os idosos foram orientados pelos agentes comunitários de saúde a levarem, na data prevista para realização da entrevista e avaliação clínica, todos os medicamentos que porventura fizessem uso, bem como suas respectivas receitas. Foi efetuado o encaminhamento imediato, à equipe responsável, de todos os indivíduos portadores de quadros de urgência odontológica. Os demais indivíduos, portadores de outras necessidades odontológicas, foram orientados a procurar o dentista de sua unidade de saúde de referência.

A variável depende deste estudo foi a autopercepção das condições de saúde bucal e as variáveis independentes foram as características sociodemográficas, necessidades odontológicas normativas, condições sistêmicas e uso de medicações. A variável dependente foi avaliada através de entrevista, utilizando o índice GOHAI, composto por 12 perguntas. Cada pergunta apresentava três respostas possíveis: "sempre", "algumas vezes" e "nunca". ${ }^{18}$ Elas receberam os escores 1, 2 e 3, respectivamente, de acordo com cada resposta, e para determinação do índice global, somava-se o escore de cada questão. ${ }^{8} \mathrm{O}$ escore de cada indivíduo podia variar de 12 a $36 .^{8}$ Dessa forma, a autopercepção do indivíduo foi classificada em "ótima" (34 a 36 pontos), "regular" (30 até 33 pontos) e "ruim" ( $<30$ pontos)..$^{14,19}$

A tabulação e análise dos dados foram realizadas no programa estatístico SPSS ${ }^{\circledast}$ para Windows ${ }^{\circledR}$, versão 15.0. Inicialmente, fez-se a análise descritiva dos dados. Posteriormente, foi conduzida uma análise bivariada para verificar a associação existente entre autopercepção e as variáveis referentes às necessidades odontológicas, características sociodemográficas, condições sistêmicas e uso de medicações, utilizando os testes Qui-quadrado de Pearson - $\mathrm{X}^{2}$ e Kruskal-Wallis. E, por fim, realizouse uma análise multivariada, baseada no método de regressão logística multinomial, utilizando as variáveis que apresentaram um valor $\mathrm{p}<0,20$ na análise bivariada. Após a regressão, o nível de significância $\alpha$ considerado foi de 0,05 .

A pesquisa foi desenvolvida de acordo com os preceitos determinados pela Resolução n ${ }^{\circ}$ 196/ 96 do Conselho Nacional de Saúde do Ministério da Saúde e de acordo com aqueles ditados pela resolução CFO 179/91 do Código de Ética Profissional Odontológica. A análise deste estudo foi feita pelo Comitê de Ética em Pesquisa da Universidade Estadual de Montes Claros (COEPUnimontes), que o aprovou, através do parecer consubstanciado ${ }^{\circ} 1079$, de 06 de julho de 2008. 
Todos os indivíduos selecionados foram devidamente informados a respeito da pesquisa e solicitou-se aos mesmos a assinatura do termo de consentimento livre e esclarecido para permissão da análise de seus dados.

\section{RESULTADOS}

Na tabela 1 está demonstrada a análise descritiva dos dados referentes às características sociodemográficas dos idosos.

Tabela 1 - Análise descritiva dos dados referentes às características sociodemográficas em uma população de idosos de Montes Claros, Minas Gerais, Brasil, 2009.

\begin{tabular}{|c|c|c|}
\hline Variáveis & Frequência (n) & Porcentagem (\%) \\
\hline \multicolumn{3}{|l|}{ Gênero } \\
\hline Masculino & 94 & $46,1 \%$ \\
\hline Feminino & 110 & $53,9 \%$ \\
\hline To tal & 204 & $100 \%$ \\
\hline \multicolumn{3}{|l|}{ Estado civil } \\
\hline Casado(a) & 94 & $46,1 \%$ \\
\hline Não casado(a) & 110 & $53,9 \%$ \\
\hline Total & 204 & $100 \%$ \\
\hline \multicolumn{3}{|l|}{ Idade } \\
\hline 60 a 69 anos & 118 & $57,8 \%$ \\
\hline 70 a 79 anos & 73 & $35,8 \%$ \\
\hline 80 anos ou mais & 13 & $6,4 \%$ \\
\hline Total & 204 & $100 \%$ \\
\hline \multicolumn{3}{|l|}{ Grupo étnico } \\
\hline Brancos & 32 & $15,7 \%$ \\
\hline Não brancos & 172 & $84,3 \%$ \\
\hline Total & 204 & $100 \%$ \\
\hline \multicolumn{3}{|l|}{ Moradia } \\
\hline Própria & 176 & $86,3 \%$ \\
\hline Alugada & 14 & $6,9 \%$ \\
\hline Cedida & 14 & $6,9 \%$ \\
\hline Total & 204 & $100 \%$ \\
\hline \multicolumn{3}{|l|}{ Escolaridade } \\
\hline Analfabetos & 141 & $69,1 \%$ \\
\hline Não analfabetos & 63 & $30,9 \%$ \\
\hline Total & 204 & $100 \%$ \\
\hline \multicolumn{3}{|l|}{ Renda } \\
\hline Até $1 / 2$ salário & 89 & $43,6 \%$ \\
\hline > 1/2 salário & 115 & $56,4 \%$ \\
\hline Total & 204 & $100 \%$ \\
\hline \multicolumn{3}{|l|}{ Já foi ao dent ista? } \\
\hline Sim & 203 & $99,5 \%$ \\
\hline Não & 1 & $0,5 \%$ \\
\hline Total & 204 & $100 \%$ \\
\hline
\end{tabular}


Tabela 1 - Análise descritiva dos dados referentes às características sociodemográficas em uma população de idosos de Montes Claros, Minas Gerais, Brasil, 2009. (Continuação)

\begin{tabular}{llc}
\hline Variáveis & Frequência (n) & Porcentagem (\%) \\
\hline Há quanto tempo? & 1 & \\
Nunca foi ao dentista & 81 & $0,5 \%$ \\
Menos de 3 anos & 122 & $39,7 \%$ \\
Há 3 anos ou mais & 204 & $59,8 \%$ \\
Total & & $100 \%$ \\
Onde? & 108 & \\
Serviço Público & 95 & $52,9 \%$ \\
Serviço Privado & 1 & $46,6 \%$ \\
Nunca foi ao dentista & 204 & $0,5 \%$ \\
Total & & $100 \%$ \\
Por quê? & 93 & \\
Confecção de prótese total removível & 54 & $45,6 \%$ \\
Outros & 56 & $26,5 \%$ \\
Dor & 1 & $27,5 \%$ \\
Nunca foi ao dentista & 204 & $0,5 \%$ \\
Total & & $100 \%$ \\
Como você avalia o atendimento? & 21 & \\
Péssimo/ruim/regular & 182 & $10,3 \%$ \\
Bom e ótimo & 1 & $89,2 \%$ \\
Nunca foi ao dentista & 204 & $0,5 \%$ \\
Total & & $100 \%$ \\
\hline
\end{tabular}

Na tabela 2 está evidenciada a análise descritiva dos dados referentes às necessidades odontológicas normativas dos indivíduos pesquisados. 
Tabela 2 - Análise descritiva dos dados referentes às necessidades odontológicas normativas em uma população de idosos de Montes Claros, Minas Gerais, Brasil, 2009.

\begin{tabular}{|c|c|c|}
\hline Variáveis & Frequência (n) & Porcentagem $(\%)$ \\
\hline \multicolumn{3}{|l|}{ Edentulismo } \\
\hline Sim & 130 & $63,7 \%$ \\
\hline Não & 74 & $36,3 \%$ \\
\hline Total & 204 & $100 \%$ \\
\hline \multicolumn{3}{|l|}{ Usa Prótese } \\
\hline Não usa prótese & 67 & $32,8 \%$ \\
\hline Usa algum tipo de prótese & 137 & $67,2 \%$ \\
\hline Total & 204 & $100 \%$ \\
\hline \multicolumn{3}{|l|}{ Necessita de prótese } \\
\hline Não necessita de prótese & 47 & $23 \%$ \\
\hline Necessita de algum tipo de prótese & 157 & $77 \%$ \\
\hline To tal & 204 & $100 \%$ \\
\hline \multicolumn{3}{|l|}{ Alterações de tecido mole } \\
\hline Ausência & 166 & $81,4 \%$ \\
\hline Presença & 38 & $18,6 \%$ \\
\hline Total & 204 & $100 \%$ \\
\hline \multicolumn{3}{|l|}{ Necessidade de cuidados imediatos } \\
\hline Ausente & 183 & $89,7 \%$ \\
\hline Presente & 21 & $10,3 \%$ \\
\hline Total & 204 & $100 \%$ \\
\hline \multicolumn{3}{|l|}{ Doença Periodontal } \\
\hline Ausência & 6 & $2,9 \%$ \\
\hline Presença & 65 & $31,9 \%$ \\
\hline Todos os sextantes excluídos & 133 & $65,2 \%$ \\
\hline Total & 204 & $100 \%$ \\
\hline \multicolumn{3}{|l|}{ Perda de Inserção Periodontal } \\
\hline Ausência & 2 & $1,0 \%$ \\
\hline Presença & 69 & $33,8 \%$ \\
\hline Todos os sextantes excluídos & 133 & $65,2 \%$ \\
\hline Total & 204 & $100 \%$ \\
\hline \multicolumn{3}{|l|}{ CPO-D } \\
\hline 8 & 1 & $0,5 \%$ \\
\hline 10 & 1 & $0,5 \%$ \\
\hline 13 & 2 & $1,0 \%$ \\
\hline 14 & 1 & $0,5 \%$ \\
\hline 15 & 1 & $0,5 \%$ \\
\hline 16 & 1 & $0,5 \%$ \\
\hline 17 & 1 & $0,5 \%$ \\
\hline 18 & 1 & $0,5 \%$ \\
\hline 19 & 1 & $0,5 \%$ \\
\hline 20 & 2 & $1,0 \%$ \\
\hline 21 & 4 & $2,0 \%$ \\
\hline 22 & 6 & $2,9 \%$ \\
\hline
\end{tabular}


Tabela 2 - Análise descritiva dos dados referentes às necessidades odontológicas normativas em uma população de idosos de Montes Claros, Minas Gerais, Brasil, 2009. (Continuação)

\begin{tabular}{|c|c|c|c|}
\hline & Variáveis & Frequência (n) & Porcentagem (\%) \\
\hline 23 & & 5 & $2,5 \%$ \\
\hline 24 & & 3 & $1,5 \%$ \\
\hline 25 & & 5 & $2,5 \%$ \\
\hline 26 & & 5 & $2,5 \%$ \\
\hline 27 & & 5 & $2,5 \%$ \\
\hline 28 & & 8 & $3,9 \%$ \\
\hline 29 & & 7 & $3,4 \%$ \\
\hline 30 & & 7 & $3,4 \%$ \\
\hline 31 & & 2 & $1,0 \%$ \\
\hline 32 & & 135 & $66,2 \%$ \\
\hline Total & & 204 & $100 \%$ \\
\hline
\end{tabular}

$\mathrm{Na}$ tabela 3, está demonstrada a análise descritiva dos dados referentes às condições sistêmicas, uso de medicações e autopercepção das condições bucais dos idosos.

Tabela 3 - Análise descritiva dos dados referentes às condições sistêmicas, uso de medicações e autopercepção das condições bucais em uma população de idosos de Montes Claros, Minas Gerais, Brasil, 2009.

\begin{tabular}{lcc}
\hline \multicolumn{1}{c}{ Variáveis } & Frequência (n) & Porcentagem (\%) \\
\hline Condição Sistêmica & & \\
Ausência de doença & 18 & $8,8 \%$ \\
Presença de doença & 186 & $91,2 \%$ \\
Total & 204 & $100 \%$ \\
Uso de medicação & & \\
Não usa medicamento & 44 & $21,6 \%$ \\
Usa medicamento & 160 & $78,4 \%$ \\
Total & 204 & $100 \%$ \\
GOHAI & & \\
Ótima & 65 & $31,9 \%$ \\
Regular & 74 & $36,3 \%$ \\
Ruim & 65 & $31,9 \%$ \\
Total & 204 & $100 \%$ \\
\hline
\end{tabular}

$\mathrm{Na}$ tabela 4, está demonstrada a análise bivariada dos dados entre a variável dependente (autopercepção das condições bucais) e as variáveis independentes nominais: características sociodemográficas, necessidades odontológicas normativas, condição sistêmica e uso de medicação nos idosos da amostra. 
Tabela 4 - Análise bivariada dos dados (variável dependente: autopercepção das condições bucaisavaliada através do índice GOHAI; variáveis independentes nominais: características sociodemo gráficas, necessidades odontológicas normativas, condição sistêmica e uso de medicação) de uma população de idosos de Montes Claros, Minas Gerais, Brasil, 2009.

\begin{tabular}{|c|c|c|c|c|c|}
\hline \multirow[t]{2}{*}{ Variáveis Independentes } & \multicolumn{3}{|c|}{ Variável Dependente } & \multirow[t]{2}{*}{ Total } & \multirow{2}{*}{$\begin{array}{c}\text { Valor } \mathrm{p} \\
\mathrm{X}^{2} \\
\end{array}$} \\
\hline & \multicolumn{3}{|c|}{ GOHAI } & & \\
\hline Gênero & Ótima & Regular & Ruim & & \\
\hline Masculino & 30 & 31 & 33 & 94 & \\
\hline$\%$ & $31,9 \%$ & $33,0 \%$ & $35,1 \%$ & $100 \%$ & \\
\hline Feminino & 35 & 43 & 32 & 110 & 0,578 \\
\hline$\%$ & $31,8 \%$ & $39,1 \%$ & $29,1 \%$ & $100 \%$ & \\
\hline Total & 65 & 74 & 65 & 204 & \\
\hline$\%$ & $31,9 \%$ & $36,3 \%$ & $31,9 \%$ & $100 \%$ & \\
\hline \multicolumn{6}{|l|}{ Estado civil } \\
\hline Casados(as) & 36 & 30 & 28 & 94 & \\
\hline$\%$ & $38,3 \%$ & $31,9 \%$ & 29,8 & $100 \%$ & \\
\hline Não casados(as) & 29 & 44 & 37 & 110 & 0,181 \\
\hline$\%$ & $26,4 \%$ & $40 \%$ & $33,6 \%$ & $100 \%$ & \\
\hline Total & 65 & 74 & 65 & 204 & \\
\hline$\%$ & $31,9 \%$ & $36,3 \%$ & $31,9 \%$ & $100 \%$ & \\
\hline \multicolumn{6}{|l|}{ Idade } \\
\hline 60 a 69 anos & 36 & 44 & 38 & 118 & \\
\hline$\%$ & $30,5 \%$ & $37,3 \%$ & $32,2 \%$ & $100 \%$ & \\
\hline 70 a 79 anos & 24 & 26 & 23 & 73 & \\
\hline$\%$ & $32,9 \%$ & $35,6 \%$ & $31,5 \%$ & $100 \%$ & 0,980 \\
\hline 80 anos ou mais & 5 & 4 & 4 & 13 & \\
\hline$\%$ & $38,5 \%$ & $30,8 \%$ & $30,8 \%$ & $100 \%$ & \\
\hline Total & 65 & 74 & 65 & 204 & \\
\hline$\%$ & $31,9 \%$ & $36,3 \%$ & $31,9 \%$ & $100 \%$ & \\
\hline \multicolumn{6}{|l|}{ Grupo étnico } \\
\hline Brancos & 10 & 13 & 9 & 32 & \\
\hline$\%$ & $31,3 \%$ & $40,6 \%$ & $28,1 \%$ & $100 \%$ & \\
\hline Não brancos & 55 & 61 & 56 & 172 & 0,832 \\
\hline$\%$ & $32,0 \%$ & $35,5 \%$ & $32,6 \%$ & $100 \%$ & \\
\hline Total & 65 & 74 & 65 & 204 & \\
\hline$\%$ & $31,9 \%$ & $36,3 \%$ & $31,9 \%$ & $100 \%$ & \\
\hline \multicolumn{6}{|l|}{ Moradia } \\
\hline Própria & 58 & 64 & 54 & 176 & \\
\hline$\%$ & $33,0 \%$ & $36,4 \%$ & $30,7 \%$ & $100 \%$ & \\
\hline Alugada & 3 & 5 & 6 & 14 & \\
\hline$\%$ & $21,4 \%$ & $35,7 \%$ & $42,9 \%$ & $100 \%$ & 0,867 \\
\hline Cedida & 4 & 5 & 5 & 14 & \\
\hline$\%$ & $28,6 \%$ & $35,7 \%$ & $35,7 \%$ & $100 \%$ & \\
\hline Total & 65 & 74 & 65 & 204 & \\
\hline$\%$ & $31,9 \%$ & $36,3 \%$ & $31,9 \%$ & $100 \%$ & \\
\hline
\end{tabular}


Tabela 4 - Análise bivariada dos dados (variável dependente: autopercepção das condições bucaisavaliada através do índice GOHAI; variáveis independentes nominais: características sociodemo gráficas, necessidades odontológicas normativas, condição sistêmica e uso de medicação) de uma população de idosos de Montes Claros, Minas Gerais, Brasil, 2009. (Continuação)

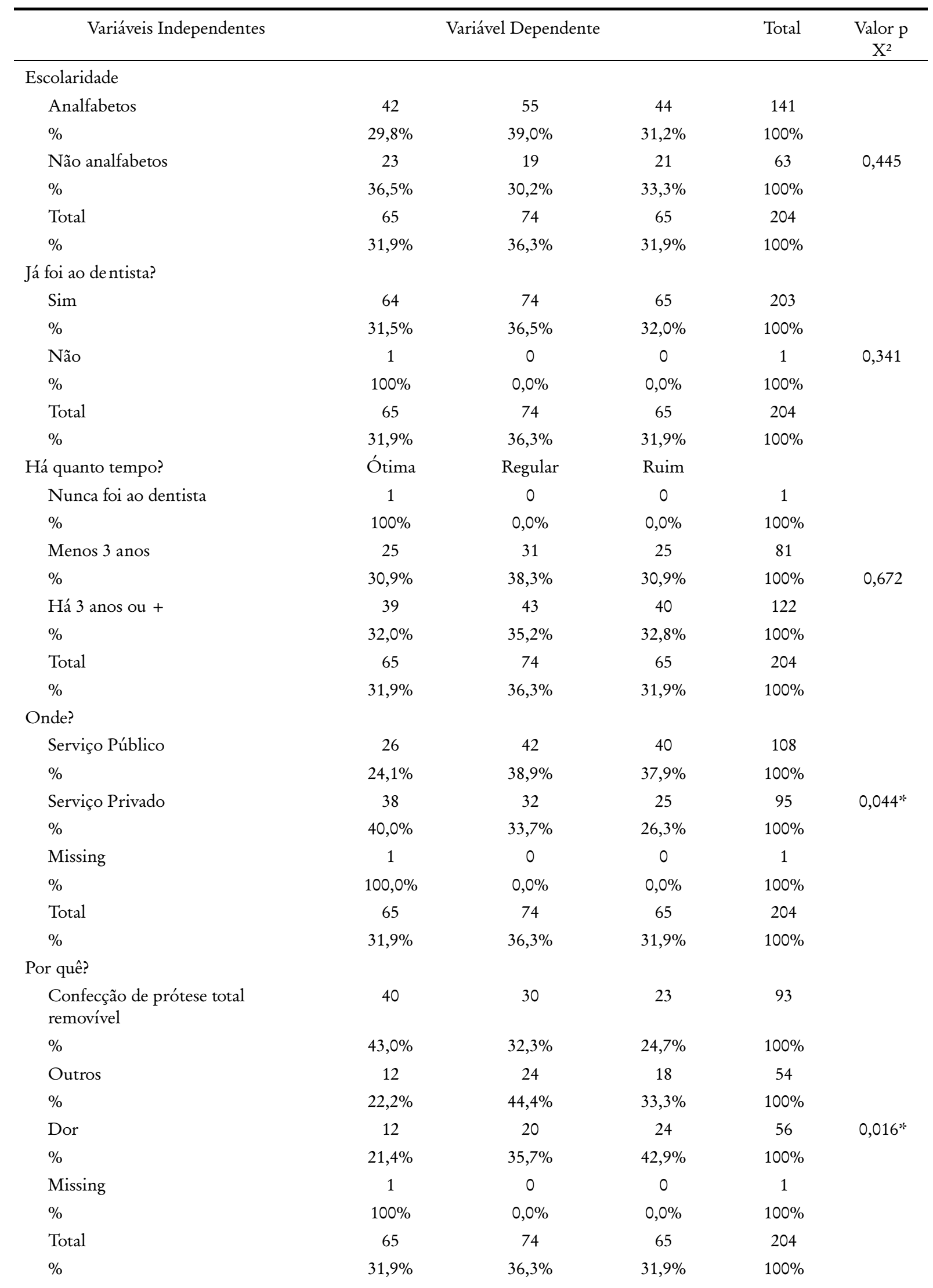


Tabela 4 - Análise bivariada dos dados (variável dependente: autopercepção das condições bucaisavaliada através do índice GOHAI; variáveis independentes nominais: características sociodemo gráficas, necessidades odontológicas normativas, condição sistêmica e uso de medicação) de uma população de idosos de Montes Claros, Minas Gerais, Brasil, 2009. (Continuação)

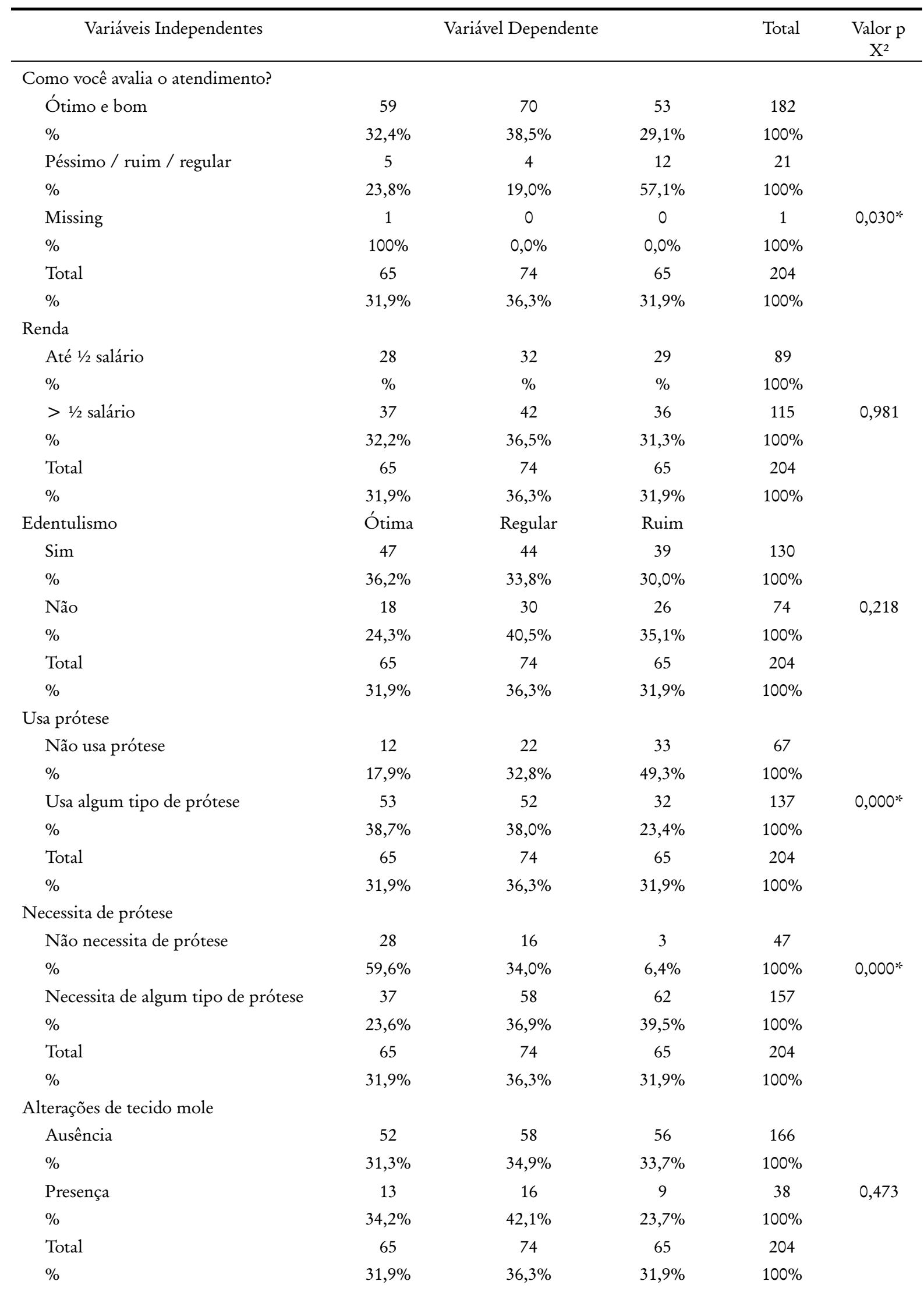


Tabela 4 - Análise bivariada dos dados (variável dependente: autopercepção das condições bucaisavaliada através do índice GOHAI; variáveis independentes nominais: características sociodemo gráficas, necessidades odontológicas normativas, condição sistêmica e uso de medicação) de uma população de idosos de Montes Claros, Minas Gerais, Brasil, 2009. (Continuação)

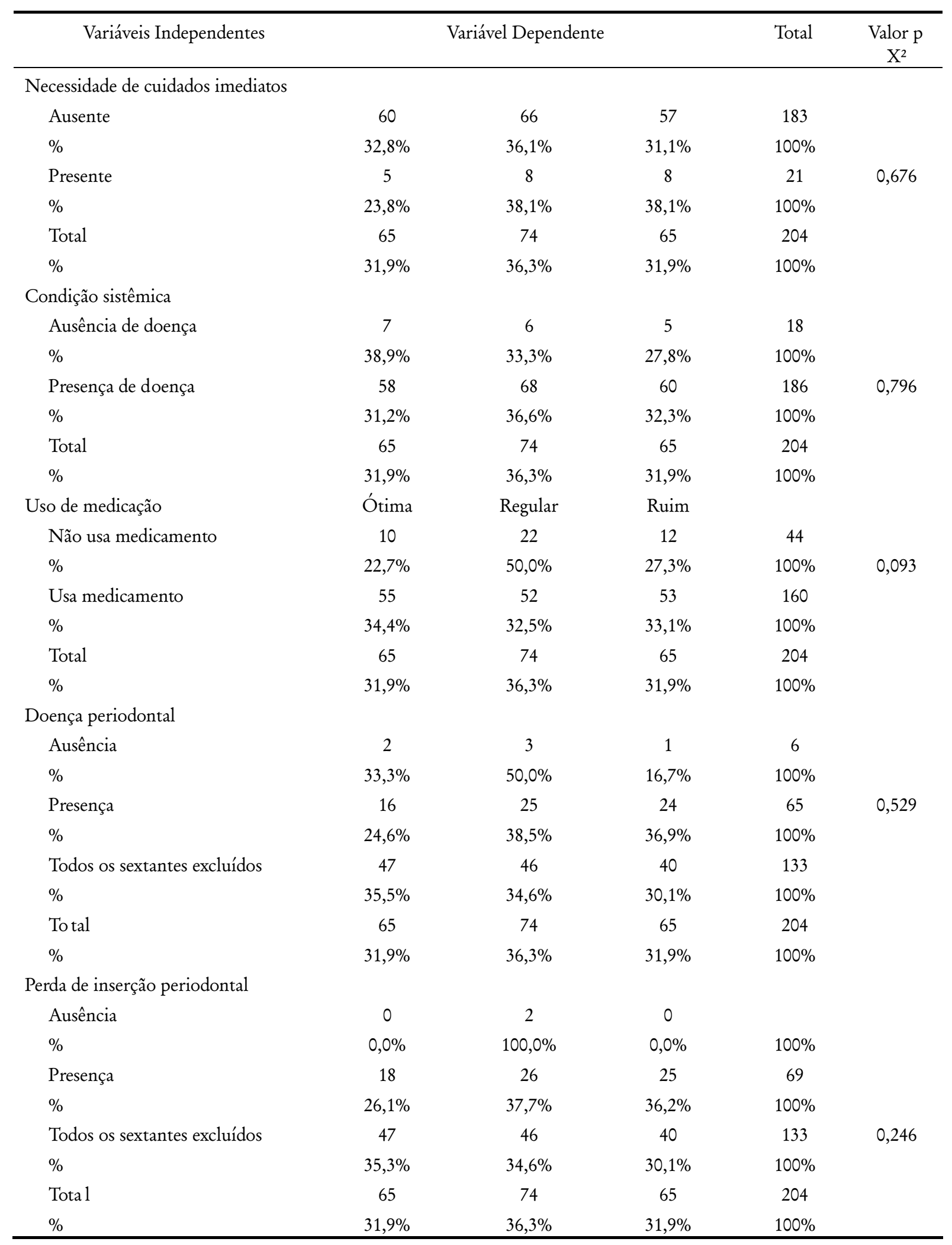

*Estatisticamente significante (Teste Qui-quadradro de Pearson - $\mathrm{X}^{2}$ ). 
Na tabela 5, está demonstrada a análise bivariada dos dados entre a variável dependente e a variável independente numérica referente à necessidade odontológica normativa (CPO-D) dos idosos.

Tabela 5 - Análise bivariada dos dados (variável dependente: autopercepção das condições bucais avaliada através do índice GOHAI; variável independente numérica: necessidade odontológica normativa - CPO-D) de uma população de idosos de Montes Claros, Minas Gerais, Brasil, 2009.

\begin{tabular}{|c|c|c|c|c|c|}
\hline Variáveis Independentes & \multicolumn{3}{|c|}{ Variável Dependente } & Total & $\begin{array}{c}\text { Valor p } \\
\text { Teste Kruskal- } \\
\text { Wallis }\end{array}$ \\
\hline & \multicolumn{3}{|c|}{ GOHAI } & & \multirow{8}{*}{0,418} \\
\hline CPO-D & Ótima & Regular & Ruim & & \\
\hline Média & 29,63 & 29,27 & 29,28 & 29,39 & \\
\hline Desvio-padrão & 5,033 & 4,911 & 4,332 & 4,754 & \\
\hline Mediana & 32 & 32 & 32 & 32 & \\
\hline Mínimo & 8 & 10 & 13 & 8 & \\
\hline Máximo & 32 & 32 & 32 & 32 & \\
\hline Intervalo de confiança $95 \%$ & $28,38-30,88$ & $28,13-30,41$ & $28,20-30,35$ & $28,73-30,04$ & \\
\hline
\end{tabular}

Na tabela 6, estão evidenciados os valores da análise multivariada entre a variável dependente e as variáveis independentes que apresentaram um valor $\mathrm{p}<0,2$ na análise bivariada.

Tabela 6 - Valores da Análise de Regressão Logística Multinomial (variável dependente: autopercepção das condições bucais - avaliada através do índice GOHAI; variáveis independentes: aquelas que apresentaram um valor $\mathrm{p}<0,2$ na análise bivariada) dos dados de uma população de idosos de Montes Claros, Minas Gerais, Brasil, 2009.

\begin{tabular}{|c|c|c|c|c|}
\hline GOHAI** & Variáveis independentes & $\begin{array}{l}\text { Odds Ratio } \\
(\mathrm{OR})^{* *}\end{array}$ & $\begin{array}{l}\text { Intervalo de confiança } \\
(95 \%)\end{array}$ & Valor $\mathrm{p}$ \\
\hline \multirow[t]{12}{*}{ Regular } & Uso de prótese & & & \\
\hline & Usa & 1,00 & - & - \\
\hline & Não usa & 1,16 & $0,48-2,81$ & 0,743 \\
\hline & Necessidade de prótese & & & \\
\hline & Não necessita & 1,00 & - & - \\
\hline & Necessita & 2,38 & $1,05-5,38$ & $0,038 *$ \\
\hline & Estado civil & & & \\
\hline & Casado(a) & 1,00 & - & - \\
\hline & Não casado(a) & 2,06 & $1,01-4,20$ & $0,046 *$ \\
\hline & Uso de medicação & & & \\
\hline & Usa & 1,00 & - & - \\
\hline & Não usa & 2,32 & $0,96-5,59$ & 0,063 \\
\hline
\end{tabular}


Tabela 6 - Valores da Análise de Regressão Logística Multinomial (variável dependente: autopercepção das condições bucais - avaliada através do índice GOHAI; variáveis independentes: aquelas que apresentaram um valor $\mathrm{p}<0,2$ na análise bivariada) dos dados de uma população de idosos de Montes Claros, Minas Gerais, Brasil, 2009. (Continuação)

\begin{tabular}{|c|c|c|c|c|}
\hline GOHAI** & Variáveis independentes & $\begin{array}{l}\text { Odds Ratio } \\
(\mathrm{OR}) * *\end{array}$ & $\begin{array}{l}\text { Intervalo de confiança } \\
(95 \%)\end{array}$ & Valor $\mathrm{p}$ \\
\hline \multirow[t]{12}{*}{ Ruim } & Uso de prótese & & & \\
\hline & Usa & 1,00 & - & - \\
\hline & Não usa & 2,35 & $1,00-5,53$ & 0,051 \\
\hline & Necessidade de prótese & & & \\
\hline & Não necessita & 1,00 & - & - \\
\hline & Necessita & 10,87 & $2,94-40,00$ & $0,000 *$ \\
\hline & Estado civil & & & \\
\hline & Casado(a) & 1,00 & - & - \\
\hline & Não casado(a) & 1,58 & $0,61-3,40$ & 0,242 \\
\hline & Uso de medicação & & & \\
\hline & Usa & 1,00 & - & - \\
\hline & Não usa & 1,05 & $0,35-2,59$ & 0,932 \\
\hline
\end{tabular}

\section{DISCUSSÃO}

Enfocando a análise descritiva dos dados, no que concerne ao gênero, resultados semelhantes foram encontrados nos Estados Unidos ${ }^{14}$ e no Brasil. ${ }^{20}$

Em relação ao estado civil, resultados discrepantes deste estudo, com uma porcentagem de idosos pesquisados casados em torno de $60 \%$, foram verificados nos Estados Unidos ${ }^{14,21}$ e no Brasil. $^{22}$

A maior parte dos idosos $(57,8 \%)$ encontravase na faixa etária compreendida entre 60 e 69 anos, corroborando os achados de outras pesquisas. ${ }^{14,23}$ Valores inferiores foram evidenciados por Matos e Lima-Costa (2006). ${ }^{24}$

No que se refere ao grupo étnico, somente $15,7 \%$ dos indivíduos se declararam como brancos. Valores superiores foram demonstrados na literatura. ${ }^{14,21}$
O estudo revelou que $86,3 \%$ dos idosos possuíam moradia própria, coincidindo com os achados de um estudo realizado no Nordeste do Brasil. ${ }^{25}$ Segundo os resultados do Projeto SB Brasil 2003, ${ }^{26} 85,96 \%$ dos idosos brasileiros tinham moradia própria. Destaca-se que os estudos envolvendo os idosos são escassos de informações referentes à moradia.

Destacando a escolaridade, a maioria dos idosos da pesquisa era analfabeta $(69,1 \%)$. A literatura revela uma grande variação de valores concernentes a essa variável em idosos..$^{22-25}$ Nos Estados Unidos, ${ }^{14}$ foi demonstrada maior prevalência de idosos com alta escolaridade, superior a 12 anos de estudo.

Observou-se que $56,4 \%$ dos idosos apresentavam renda per capita superior a $1 / 2$ salário mínimo (salário vigente à época - novembro de 2008 - R\$ 415,00). Matos \& Lima-Costa, ${ }^{24}$ ao avaliarem a saúde bucal de idosos da Região 
Sudeste do Brasil, encontraram que 39,6\% dos pesquisados tinham renda domiciliar percapita igual ou superior a $1 \frac{1}{2}$ salário mínimo (salário vigente à época - agosto de $2006-\mathrm{R} \$ 350,00)$.

No que diz respeito ao acesso aos serviços odontológicos, o estudo demonstrou que $99,5 \%$ dos idosos já foram ao dentista alguma vez. Achados semelhantes foram demonstrados em outras avaliações. ${ }^{24,25} \mathrm{De}$ acordo com os achados do Projeto SB Brasil 2003, ${ }^{26}$ 94,19\% dos idosos pesquisados consultaram pelo menos uma vez com um dentista.

Verificou-se que $59,8 \%$ dos idosos do estudo foram ao dentista há três anos ou mais. Ritter et al..$^{22}$ encontraram que $52 \%$ dos idosos avaliados tinham ido ao dentista há mais de três anos. De acordo com os resultados do Projeto SB Brasil $2003,{ }^{26} 65,69 \%$ dos idosos pesquisados consultaram com um dentista há três anos ou mais. No trabalho de Matos e Lima-Costa, ${ }^{24}$ 69,6\% dos idosos tinham ido ao dentista há trêsanos ou mais. O valor encontrado por Santos et al. ${ }^{25}$ foi de $37,9 \%$.

Em relação ao local onde foi realizada a última consulta odontológica, $52,9 \%$ dos idosos investigados realizaram-na no serviço público. Dado semelhante foi obtido em outra avaliação. ${ }^{25}$ Valor inferior foi observado no Projeto SB Brasil 2003. ${ }^{26}$

No que se refere ao motivo da última consulta odontológica, 45,6\% dos idosos relataram ter procurado o serviço para confecção de prótese total removível. No estudo de Ritter et al., ${ }^{22} 41 \%$ dos idosos afirmaram que o motivo da última consulta foi confecção, adequação ou conserto de próteses. O principal motivo encontrado na pesquisa de Santos et al..$^{25}$ foi a consulta de rotina (37,9\%). Já no Projeto SB Brasil 2003, ${ }^{26}$ o principal motivo da última consulta foi a dor $(48,12 \%)$.

A maioria dos idosos (89,2\%) avaliou o último atendimento recebido como bom ou ótimo. Frequências menores foram verificadas em outros trabalhos..$^{25,26}$

No que concerne às necessidades odontológicas normativas, $63,7 \%$ dos idosos eram edêntulos. Resultado similar foi encontrado por outros autores. ${ }^{25}$ Menores prevalências foram encontradas nos Estados Unidos ${ }^{14}$ e no Brasil. ${ }^{27}$

Observou-se que $67,2 \%$ dos idosos usavam algum tipo de prótese. Valores superiores foram encontrados por Ritter et al. ${ }^{22} \mathrm{e}$ Bonan et $\mathrm{al} .{ }^{20} \mathrm{No}$ estudo de Santos et al. ${ }^{25}$ um valor inferior a esse foi verificado.

Foi observado que $77 \%$ dos idosos necessitavam de algum tipo de prótese. Frequência menor foi obtida por Santos et al..$^{25}$

Destacando as alterações de tecido mole dos idosos pesquisados, $81,4 \%$ não apresentavam nenhuma alteração. No Sul do Brasil, ${ }^{27}$ pesquisadores encontraram uma prevalência de $59,3 \%$.

A presente pesquisa demonstrou que $89,7 \%$ dos idosos não necessitavam de cuidados odontológicos imediatos. Nos Estados Unidos, ${ }^{14}$ $72,11 \%$ dos idosos investigados necessitavam de tratamento dentário. No estudo de Matos \& Lima-Costa, ${ }^{24} 50,2 \%$ dos idosos avaliados referiram necessidade de tratamento odontológico.

Nos idosos pesquisados, a prevalência de doença periodontal foi de $31,9 \%$. Valor semelhante foi obtido no Projeto SB Brasil $2003^{26}$ e quantia superior foi verificada por Henriques et al. ${ }^{19}$ Apenas $2,9 \%$ dos idosos da pesquisa apresentavam todos os sextantes sadios. Valor um pouco maior $(7,89 \%)$ foi encontrado em idosos brasileiros. ${ }^{26}$ No presente estudo, houve grande frequência de indivíduos que tiveram todos os sextantes excluídos na avaliação do İndice Periodontal Comunitário - CPI $(65,2 \%)$. Resultados similares foram encontrados em outros trabalhos. ${ }^{26,27}$

Seguindo os mesmos critérios de avaliação da doença periodontal, ao se pesquisar a perda de inserção periodontal, verificou-se uma grande porcentagem de idosos $(65,2 \%)$ que tiveram todos os sextantes excluídos na investigação do Índice de Perda de Inserção Periodontal (PIP). A presença de perda de inserção foi observada em 
$33,8 \%$ dos indivíduos. Em outra pesquisa, ${ }^{28}$ a frequência observada foi de $55,8 \%$. Ressalta-se que foram encontrados poucos estudos avaliando a perda de inserção periodontal em idosos.

Os idosos da presente pesquisa apresentaram um índice CPO-D elevado, com média de 29,39, cuja maior parte foi atribuída ao componente perdido. Esse resultado corrobora o valor médio encontrado por outros autores. ${ }^{25}$

No que se refere à condição sistêmica, 91,2\% dos idosos apresentavam alguma doença crônica. Dado semelhante foi encontrado na literatura ${ }^{23}$. No Japão, ${ }^{29} 64,2 \%$ dos idosos avaliados apresentavam doenças sistêmicas. Nos Estados Unidos, ${ }^{3} 75 \%$ das pessoas com mais de 65 anos possuíam alguma doença crônica.

Foi verificado no estudo que a maior parte dos idosos $(78,4 \%)$ fazia uso de algum medicamento. Resultado semelhante foi verificado em outra pesquisa. ${ }^{3}$ Frequência diferente foi verificada por outros pesquisadores $(58 \%){ }^{20}$

Enfocando a autopercepção, a maioria dos idosos (36,3\%) autopercebeu a condição bucal como regular e $31,9 \%$ autoperceberam como ruim. Valores semelhantes foram obtidos no Brasi1 ${ }^{23}$ e nos Estados Unidos. ${ }^{14} \mathrm{~A}$ maioria dos idosos investigados por Santos et al. ${ }^{25}$ no Brasil apresentou baixa autopercepção em saúde bucal.

Destacando os valores médios do GOHAI demonstrados na literatura internacional e nacional, trabalho feito nos Estados Unidos ${ }^{21}$ obteve valores do índice de 33,36 e 31,77 em duas amostras pesquisadas, qualificando a autopercepção das condições bucais dos idosos como regular. Dados similares foram observados no Brasil. ${ }^{8}$ Valores discrepantes, característicos de uma autopercepção de saúde bucal ruim, foram observados por outros autores. ${ }^{19,20,25}$

$\mathrm{Na}$ análise bivariada, observou-se associação estatisticamente significante $(p<0,05)$ entre a variável dependente autopercepção das condições bucais e as seguintes variáveis independentes: onde? (onde foi realizada a última consulta odontológica); por quê? (motivo da última consulta odontológica); como você avalia o atendimento? (avaliação do último atendimento odontológico recebido); uso de prótese superior, uso de prótese inferior, uso de prótese (qualquer tipo de prótese superior e/ou inferior); necessidade de prótese superior, necessidade de prótese inferior e necessidade de prótese (qualquer tipo de prótese superior e/ou inferior). A maioria dos idosos que realizaram a última consulta odontológica no serviço público, cujo motivo da última consulta foi a confecção de prótese total removível, que avaliaram o último atendimento como ótimo ou bom, que não usavam prótese superior e/ou inferior e necessitavam de prótese superior e/ou inferior apresentaram baixos escores no índice GOHAI, característicos de uma baixa autopercepção das condições bucais.

Atchison \& Dolan, ${ }^{14}$ nos Estados Unidos, observaram associação significante entre autopercepção das condições bucais e idade, gênero, raça, estado civil, escolaridade, renda familiar anual, uso de prótese removível, necessidade atual de tratamento dentário e número de dentes em idosos. De acordo com os resultados encontrados por essas autoras, os idosos com alto status socioeconômico tiveram melhor autopercepção, já que os indivíduos que tinham melhor educação eram brancos e possuíam alta renda anual obtiveram maiores escores no GOHAI. Ainda no que diz respeito a esses resultados, destacando-se as características clínicas, os escores do GOHAI foram maiores nos idosos que possuíam entre 21 e 32 dentes, não usavam prótese removível e não necessitavam de tratamento dentário autodeclarado.

Kressin et al., ${ }^{21}$ nos Estados Unidos, encontraram associação significante entre autopercepção das condições bucais e idade, raça, estado civil, escolaridade e renda nos indivíduos pesquisados. Os idosos que possuíam mais de 85 anos, não eram brancos, não eram casados, possuíam baixa escolaridade e baixa renda obtiveram baixos escores no GOHAI.

$\mathrm{Na}$ China, ${ }^{30}$ um estudo verificou que a consulta recente ao cirurgião-dentista foi 
determinante para uma baixa autopercepção da saúde bucal em idosos. Na França, ${ }^{31}$ foi demonstrado que idosos com pouca escolaridade e baixa renda tinham baixa autopercepção das condições bucais. Segundo os autores desse trabalho, idosos que usavam uma ou mais próteses removíveis apresentaram pior autopercepção quando comparados aos que não faziam uso de próteses.

Rodrigues, ${ }^{32}$ no Brasil, observou associação significante entre autopercepção das condições bucais e estado civil, escolaridade, estilo de vida (vivia sozinho, com cuidador, com esposo/a, com outra pessoa) e tipo de moradia (habitação comunitária ou própria). Os idosos solteiros, analfabetos, que moravam com outra pessoa e viviam em habitação comunitária apresentaram escores mais baixos no GOHAI, portanto tiveram uma pior autopercepção. $\mathrm{O}$ autor não verificou associação entre o índice e gênero, idade, renda, saúde geral, última visita ao dentista, uso de prótese superior e inferior nos indivíduos pesquisados.

Tiago $^{23}$ encontrou associação significante entre autopercepção de saúde bucal e idade. $\mathrm{O}$ autor destacou que as pessoas com mais idade tenderam a ter baixa percepção na dimensão física/funcional do GOHAI; em relação às dimensões psicológica/ psicossocial e dor/desconforto, observou-se grande variação de idade entre os idosos que tiveram uma boa ou alta autopercepção. Esse autor verificou ainda que não houve associação entre GOHAI e gênero, estado civil e escolaridade nos idosos.

Henriques et al., ${ }^{19}$ ao avaliarem idosos no Estado de São Paulo, Brasil, observaram associação não-significante entre GOHAI e doença periodontal, cárie radicular e uso de próteses.

Santos et $a l .^{25}$ encontraram associação significante entre GOHAI e necessidade de prótese. A maior parte dos idosos que necessitava de prótese teve baixa autopercepção das condições bucais. Não foi encontrada associação entre o índice e uso de prótese.

$\mathrm{Na}$ pesquisa de Bonan et al., ${ }^{20}$ os autores verificaram associação significante entre GOHAI e uso de medicação. Os idosos que não utilizavam medicação apresentaram média do índice mais alta, qualificando assim como moderada a autopercepção das condições bucais daqueles indivíduos.

$\mathrm{Na}$ análise multivariada, foi observada associação estatisticamente significante $(\mathrm{p}<0,05)$ entre a variável dependente "autopercepção das condições bucais" e as seguintes variáveis independentes: necessidade de prótese e estado civil. Os idosos que necessitavam de prótese tinham uma chance de 2,38 vezes de autoperceber sua condição bucal como regular do que autopercebê-la como ótima, e uma chance de 10,87 vezes de perceber essa condição como ruim em comparação à melhor autoavaliação. Já os indivíduos que não eram casados tinham uma chance de 2,06 vezes de autoperceber sua condição bucal como regular do que autopercebê-la como ótima. Por conseguinte, os idosos que necessitavam de algum tipo de prótese dentária e não possuíam cônjuge apresentaram baixa autopercepção da saúde bucal.

No estudo de Kressin et al., ${ }^{21}$ os autores verificaram, através de análise de regressão múltipla, haver associação significante entre autopercepção das condições bucais, renda e estado civil em idosos americanos. De acordo com os resultados, a renda e estado civil, em um dos grupos avaliados, chegou a explicar $6 \% \mathrm{da}$ variância do índice GOHAI.

$\mathrm{Na}$ pesquisa de Rodrigues, ${ }^{32}$ o autor demonstrou, através de análise de regressão múltipla, que os fatores de risco para baixos valores do GOHAI foram: estado civil e tipo de moradia. Logo, os indivíduos solteiros e que moravam em habitação coletiva possuíam maior risco de apresentar uma baixa autopercepção das condições bucais.

Vale a pena ressaltar que os resultados encontrados neste estudo são representativos do grupo etário local, não podendo ser generalizados para todos os idosos da cidade de Montes Claros, MG. 


\section{CONCLUSÕES}

De maneira geral, percebe-se que a maior parte dos idosos da amostra possuía uma condição socioeconômica satisfatória, com exceção da baixa escolaridade; apresentava necessidades odontológicas normativas, destacando-se o edentulismo, uso e necessidade de prótese, doença periodontal e perda de inserção periodontal; tinha alguma doença sistêmica; usava medicação e autopercebeu a condição bucal como regular.

A maior parte dos idosos que realizaram a última consulta odontológica no serviço público, cujo motivo da última consulta foi a confecção de prótese total removível, que avaliaram o último atendimento como ótimo ou bom, que não usavam prótese superior e/ou inferior e

\section{REFERÊNCIAS}

1. Werner $\mathrm{CW}$ et al. Odontologia geriátrica. Revista da Faculdade de Odontologia de Lins 1998; 11: $62-70$.

2. Larocca CD, Jahnigen DW. Medical history and risk assessment. Dent. Clin. N. Amer. 1997; 41(4): 669-79.

3. Cormack E. A saúde oral do idoso. 2000. Disponível em: URL: http:// www.odontologia.com.br/artigos/ geriatria.html.

4. Frare SM et al. Terceira idade: quais os problemas bucais existentes? Ver Assoc Paul Cir Dent 1997; 51(6): 573-6.

5. Rosa LB et al. Odontogeriatria - a saúde bucal na terceira idade. RFO 2008 mai./ago; 13(2): 82-86.

6. Pucca Junior GA. Saúde bucal do idosos: aspectos sociais e preventivos. In: Papaléo Netto M. Gerontologia. São Paulo: Atheneu; 1996.

7. Paulander JA et al. Association between level of education and oral health status in 35-, 50-, 65 and 75-years-old. J Clin Periodontol 2003; 30: 697-704.

8. Silva SRC, Castellanos-Fernandes RA. Autopercepção das condições de saúde bucal por idosos. Rev. Saúde Pública 2001; 35: 349-55.

9. Ofstedal MB et al. Self-assessed health expectancy among older Asians: a comparison of Sullivan and multistate life table methods. Ann Arbor: Population Studies Center; 2002. necessitavam de prótese superior e/ou inferior apresentou baixa autopercepção das condições bucais, com associação estatisticamente significante na análise bivariada.

$\mathrm{Na}$ análise multivariada, foi verificado que os idosos que necessitavam de algum tipo de prótese dentária e não possuíam cônjuge apresentaram maiores chances de ter uma baixa autopercepção das condições bucais.

Foi observado neste estudo que a autopercepção de saúde bucal dos idosos foi condizente com a avaliação profissional. Os resultados obtidos evidenciam a necessidade de uma maior oferta de serviços de promoção de saúde, prevenção de agravos e de atendimento curativo/reabilitador que sejam destinados a esse grupo populacional.

10. Steele JG et al. Clinical factors related to reported satisfaction with oral function amongst dentale older adults in England. Community Dent Oral Epidemiol 1997; 25: 143-9.

11. Slade GD, Spencer AJ. Social impact of oral conditions among older adults. Austr Dent J 1994; 39: 358-64.

12. Reisine ST, Bailit HL. Clinical oral health status and adult perceptions of oral health. Soc Sci Med 1980; 14: 597-605.

13. Lourenço RA, Veras RP. Mini-Exame do Estado Mental: características psicométricas em idosos ambulatoriais. Rev Saúde Pública 2006; 40(4): 712-9.

14. Atchison KA, Dolan TA. Development of the Geriatric Oral Health Assessment Index. J Dent Educ 1990; 54: 680-7.

15. Brasil. Ministério da Saúde. Projeto SB 2000: condições de saúde bucal da população brasileira: manual do examinador. Brasília: Ministério da Saúde; 2001.

16. Organização Mundial da Saúde. Levantamento epidemiológico básico de saúde bucal. 3. Ed. São Paulo: Santos; 1991.

17. Landis JR, Koch GG. A medição da concordância do observador para dados por categoria. Biometrics 1977; 33-159 apud Gordis L. Epidemiologia. 2. ed. Rio de Janeiro: Revinter; 2004. 
18. Pinto VG. Saúde Bucal Coletiva. 4. ed. São Paulo: Santos; 2000.

19. Henriques $\mathrm{C}$ et al. Autopercepção das condições de saúde bucal de idosos do município de Araraquara - SP. Cienc Odontol Bras 2007; 10(3): 67-73.

20. Bonan PRF et al. Condições bucais e de reabilitação insatisfatórias dissociadas da percepção de qualidade de vida em idosos institucionalizados e não-institucionalizados. Rev. Odonto ciênc. 2008; 23(2): 115-119.

21. Kressin NR, Atchison KA, Miller DR. Comparing the impact of oral disease in two populations of older adults: application of the Geriatric Oral Health Assessment Index. J Public Health Dent 1997; 57: 224-32.

22. Ritter $\mathrm{F}$ et al. Condições de vida e acesso aos serviços de saúde bucal de idosos da periferia de Porto Alegre. Boletim da Saúde 2004 jan./jun.; 18(1).

23. Tiago ED. Autopercepção em relação à condição clínica de saúde bucal em idosos nãoinstitucionalizados portadores de próteses totais. Dissertação (Mestrado em Ciências da Saúde) - Universidade de Brasília, UnB, Brasília, DF; 2006.

24. Matos DL, Lima-Costa MF. Auto-avaliação da saúde bucal entre adultos e idosos residentes na Região Sudeste: resultados do Projeto SB-Brasil, 2003. Cad. Saúde Pública 2006 ago.; 22(8): 16991707.
25. Santos FB et al. Autopercepção em saúde bucal de idosos em unidades de saúde da família do distrito sanitário III de João Pessoa-PB. Arquivos em odontologia 2007 abri./jun.; 43(2): 23-32.

26. Brasil. Ministério da Saúde. Projeto SB Brasil 2003: Condições de Saúde Bucal da População Brasileira: resultados principais. Brasília: Ministério da Saúde; 2004. Disponível em: URL: http://www.cfo.org.br/download/pdf/ relatorio_sb_brasil_2003.pdf.

27. Mesas AE et al. Condições de saúde bucal de idosos de comunidade urbana de Londrina, Paraná. Rev. Bras. Epidemiol 2006; 9(4): 471-80.

28. Bianco VC et al. O impacto das condições bucais na qualidade de vida de pessoas com 50 um mais anos de vida. Revista Ciência \& Saúde Coletiva; 2008.

29. Umino M, Nagao M. Systemic diseases in elderly dental patients. Int Dent J 1993; 43(3): 213-8.

30. Wong CMM et al. Translation and Validation of the Chinese Version of GOHAI. J Public Health Dent 2002; 62(2): 78-83.

31. Tubert-Jeannin $\mathrm{S}$ et al. Validation of an oral quality of life index (GOHAI) in France. Community Dent Oral Epidemiol 2003; 31: 275-284.

32. Rodrigues CK. Autopercepção de Saúde Bucal em Idosos. Dissertação (Mestrado em Saúde Coletiva) - Faculdade de Odontologia de Piracicaba, da Universidade Estadual de Campinas, Unicamp, Piracicaba, SP; 2005. 
